\title{
A New Use for the Amplatzer Duct Occluder Device
}

\author{
Carlos A. C. Pedra, Luciano N. Sousa, Carlo B. Pilla, Valmir F. Fontes
}

São Paulo, SP - Brazil

\begin{abstract}
We report a case in which the Amplatzer device for percutaneous occlusion of ductus arteriosus was successfully used for occluding a large systemic-pulmonary collateral vessel in a patient who had previously undergone surgery for correction of pulmonary atresia and ventricular septal defect (Rastelli technique), and was awaiting the change of a cardiac tube. In the first attempt, the device embolized to the distal pulmonary bed and, after being rescued with a Bitome, it was appropriately repositioned with no complications and with total occlusion of the vessel.
\end{abstract}

Based on the publication of results of convincing experimental studies ${ }^{1}$, the new Amplatzer device for percutaneous occlusion of ductus arteriosus (AGA Medical Corporation, Golden Valley, MN, USA) has been shown to be safe and highly effective in clinical practice ${ }^{2-5}$. Due to the versatility of the implantation system and the characteristics of the prosthesis, it has also been used for occlusion of other defects and vascular malformations, such as coronary and pulmonary arteriovenous fistulae, systemic-pulmonary shunts (Blalock-Taussig), venovenous collaterals after Glenn surgery, tubes, fenestrated Fontan, and others ${ }^{6-8}$. We report a case in which this device was successfully used for closure of a large systemic-pulmonary collateral vessel originating from the descending aorta.

\section{Case Report}

The patient is a 17-year-old male who underwent surgical repair of pulmonary atresia, ventricular septal defect, and systemic-pulmonary collaterals at the age of 7 years. The collaterals were focalized, the ventricular septal

Instituto Dante Pazzanese de Cardiologia

Mailing address: Carlos A. C. Pedra - Instituto Dante Pazzanese de Cardiologia - Av. Dr. Dante Pazzanese, 500 - 04012-180 - São Paulo, SP - Brazil - Email: vffontes@uol.com.br

English version by Stela Maris C. e Gandour defect was occluded with a patch of bovine pericardium and an 18-mm Dacron tube graft, which was used to reestablish the continuity between the right ventricle and the pulmonary artery (Rastelli technique). The patient complained of mild fatigue on exertion, and on physical examination an ejective systolic murmur in the middle left sternal margin and a continuous murmur in the dorsum could be heard. On chest X-ray, slight cardiomegaly and increased pulmonary flow to the right middle and lower lobes were observed. The electrocardiogram showed sinus rhythm and biventricular hypertrophy. The twodimensional Doppler color flow echocardiogram showed signs of obstruction of the tube with rectification of the ventricular septum during systole. Right ventricular pressure was estimated in approximately $3 / 4$ of the arterial blood pressure through tricuspid reflux. Moderate pulmonary insufficiency also existed. The patient was referred for cardiac catheterization for diagnostic complementation, and the examination, performed with the patient under local anesthesia, showed the following pressure levels (in $\mathrm{mm} \mathrm{Hg}$ ): right atrium, 9; right ventricle, 90/10; pulmonary artery, 30/11; left ventricle, 110/12; and aorta, 110/ 60 . Right ventriculography revealed obstruction of the tube in its proximal portions, next to the right ventricular musculature, and mild to moderate systolic dysfunction. The aortogram, followed by selective injection in the vessel, showed a large collateral vessel originating from the right lateral portion of the descending aorta with a sinuous trajectory, which initially headed upward and then bent downward. This collateral vessel irrigated part of the middle and lower lobes of the right lung, communicating with the native pulmonary bed. A stenotic point after the angulation site was identified. Due to high pressure levels in the right ventricular cavity accompanied by systolic dysfunction, surgery to exchange the tube was indicated, preceded by elective occlusion of the systemic-pulmonary collateral vessel via the percutaneous route. The possibility of stent implantation inside the tube was discarded, because the stenotic point was very close to the ventricular musculature. Due to the dimensions of and high 
flow in the collateral, and the high thrombogenic potential of the Amplatzer duct occluder device, we chose to use it to close the vessel. In a second catheterization, also performed with local anesthesia , a 6 Fr sheath was placed in the right femoral artery, followed by administration of heparin (5,000 IU). The collateral vessel was selectively catheterized with the aid of a hydrophilic-coated guidewire, and a right coronary Judkins catheter was distally positioned beyond the stenotic point. A 0.035 " super stiff guidewire (Cook) was passed inside the Judkins catheter, replaced by a long Mullins sheath, which was part of the kit used for occlusion of ductus arteriosus, and was advanced with no difficulty through the collateral vessel and distally positioned beyond the curvature. After being charged according to the usual instructions, an 8-6 Amplatzer device was advanced through the sheath with no difficulty as far as the narrowing point. After test

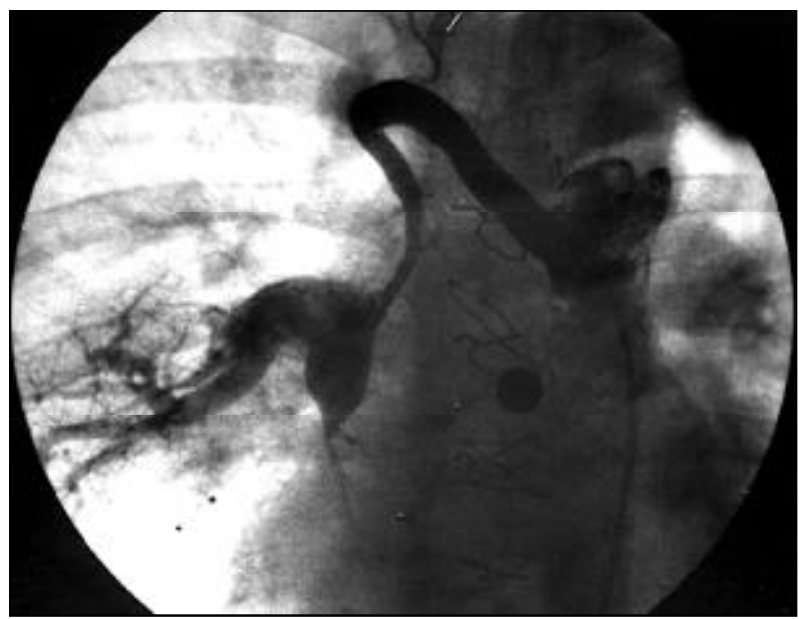

Fig. 1 - Large collateral vessel originating from the descending aorta. The closest point to the aorta measured $11 \mathrm{~mm}$ and the most stenotic one, after the angulation site, $4.5 \mathrm{~mm}$. Note the 8-6 Amplatzer duct occluder device embolized in the distal pulmonary bed. The distal retention skirt is located upwards.

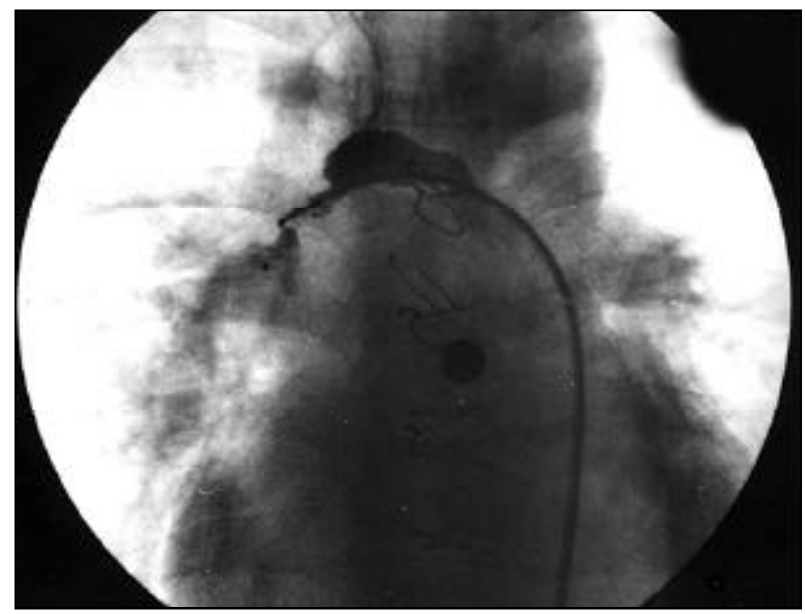

Fig. 2 - Location of the prosthesis right before delivery. Note the accordion or pleating phenomenon in the vessel due to the presence of the system inside it. injections into the lateral arm of the long sheath to check its position, the Amplatzer device was delivered, but its immediate migration to the distal pulmonary bed occurred (fig. 1). Retrospectively, we observed that the site of delivery was beyond the angulation and stenotic point. Due to the presence of the sheath inside the collateral vessel, a distortion in the vessel trajectory occurred, with the occurrence of the accordion or pleating phenomenon (fig. 2). Maintaining the sheath at the same site, a $3 \mathrm{Fr}$ Bitome (Cook) was advanced into the vessel, where the device had embolized. After several attempts, rescue through a bite from the device's central pin by the teeth of the Bitome was performed on the side of the disc of distal retention (retained upwards). The prosthesis was then pulled inside the sheath and removed from the body, and no damage in its structure was observed. After recharging, the prosthesis was advanced again through the sheath and delivered to the correct site, with immediate occlusion of the collateral vessel (fig. 3). Fluoroscopy lasted 30 minutes, and the procedure lasted 2 hours. On the following day, the patient underwent surgical replacement of the tube, being discharged from the hospital after 1 week. Surgery and catheterization were uneventful.

\section{Discussion}

In the present case, the reasons for indicating elective percutaneous occlusion of the systemic-pulmonary collateral vessel were the following: reduction in increased pulmonary blood flow and volume overload of the left chambers; abolition of the left-right shunt and consequent significant reduction in pulmonary venous return, providing a clearer surgical field during surgical replacement of the tube under extracorporeal circulation; elimination of the possible systemic blood flow steal during surgery, minimizing the risk of neurologic compli-

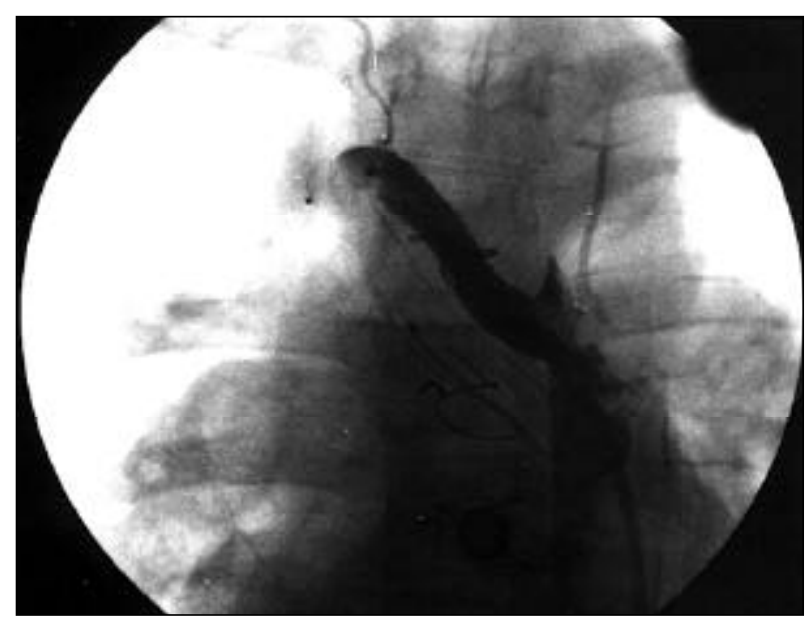

Fig. 3 - Adequate final location of the prosthesis after rescuing and repositioning. No residual shunt is present. 
cations ${ }^{9,10}$. In addition, surgical time was reduced, because the surgeon did not have to extend his plane of dissection as far as the posterior mediastinum to locate and ligate the collateral during surgery. In a patient with previous cardiac surgery, this maneuver, which is by itself very hard, is even more difficult due to the presence of fibrosis and scar tissue. Because our patient's collateral vessel communicated with the native pulmonary bed, its occlusion in the appropriate place would not cause pulmonary infarction. Elective occlusion of these collaterals, before or after surgical procedures, is probably the most common procedure performed in a hemodynamics laboratory ${ }^{11}$, and it reflects the differentiated work of cooperation between the surgeon and the interventionist in managing complex congenital heart diseases. Several devices have been used to occlude these vessels, the Gianturco coils being used most frequently ${ }^{11}$. The index of total occlusion with these intravascular prostheses is approximately $90 \%$, and residual shunts are more commonly found in large-caliber and high-flow vessels, as the one here described. The complications are not common and are as follows: embolization to the lungs or systemic circulation is 1 possibility, because the delivery system is not controlled; low fever after occlusion is not infrequent; and intravascular hemolysis is sometimes reported when occlusion is incomplete ${ }^{11}$.

The Amplatzer occluder device (AGA Medical Corporation, Golden Valley, MN, USA) for percutaneous closure of ductus arteriosus is a self-expanding device shaped like a mushroom, and consisting of a 0.004 " metallic mesh of nitinol, which has an intrinsic memory. A retention disc or skirt, whose diameter is $4 \mathrm{~mm}$ larger than that of the device, exists. Flaps of polyester fibers are sewn to the metallic mesh in the inner portion of the prosthesis, which accounts for inducing rapid local thrombosis. A stainless steel delivery cable connects to a female pin in the device through a screw mechanism. A long Mullins sheath, a plastic charger, and a tubular device to rotate the delivery cable complete the implantation kit. The prosthesis is available in 5 combinations of diameters in $7 \mathrm{~mm}$ or $8 \mathrm{~mm}$ lengths. The profile of the sheath ranges form $5 \mathrm{Fr}$ to $7 \mathrm{Fr}$, according to the diameter selected ${ }^{1-5}$. A great technical advantage of this implantation system is the possibility of rescuing the prosthesis inside the sheath, if its location is not adequate, before its final delivery, and this procedure is totally controlled by the operator. The world clinical experience and that in Brazil show a 100\% efficacy for occlusion of ductus arteriosus with very low indices of complications ${ }^{2-5}$. Due to the favorable characteristics of the system, this prosthesis has also been used to occlude other vascular structures ${ }^{6-8}$. In the case here reported, the
Amplatzer prosthesis was selected for occlusion due to its high thrombogenic potential and also because the vessel in question was large in caliber and high in blood flow. We understand that implantation of multiple coils could also have been tried for the same purpose ${ }^{11}$. A lower cost would be an advantage of this latter type of approach, even though it provides lower indices of occlusion. No cost restrictions existed in this case, because the Amplatzer prosthesis was covered by the patient's medical insurance, and we tried to obtain maximum clinical efficacy. The low profile of the sheath required for implantation made its use in the artery possible. The entire system was very flexible allowing the curvature of the vessel in question. Even though assessment of the location of the prosthesis was possible prior to final delivery through injections of contrast medium via the lateral arm of the long sheath, the delivery was performed too distally with immediate embolization. Due to the already known tendency of systemic-pulmonary collaterals to pleat when undergoing percutaneous handling, in association with the anatomic distortions caused by the sheath and the delivery cable inside the vessel, the authors misinterpreted the correct site for delivery. To avoid causing a pulmonary infarction, because of the rapid induction of local thrombosis by the device, we chose to rescue it immediately. According to the world literature ${ }^{6}$, the occurrence of embolization of this prosthesis is very rare; therefore, the choice of an efficient system or catheter for rescuing it was difficult. Based on personal experience with a case in The Hospital for Sick Children in Toronto, $\mathrm{Canada}^{6}$, with a successful rescue of an Amplatzer device that had embolized to the descending aorta after an attempt to close a ductus arteriosus, we chose to use a Bitome for endomyocardial biopsy. Coupling of the Bitome's teeth with the central pin of the prosthesis in the retention skirt required greater perseverance and patience in the technician than proper technical skill. Because the device was collapsible and had intrinsic memory, it was pulled inside the sheath with no damage to its metallic structure. Consequently, the same device could be used in a second attempt at implantation and vascular occlusion in the catheterization room, but, this time, with success.

In conclusion, the Amplatzer device for ductus arteriosus has very versatile characteristics, which enable it to be used for closure of other vascular structures, such as the systemic-pulmonary collateral vessel here reported. The presence of the sheath-cable-prosthesis set inside the target vessel may distort its anatomy, confusing the delivery site. In case of embolization, the use of a Bitome seems an appropriate resource for rescue. 


\section{References}

1. Sharafundin MJ, GuX, Titus JL, et al. Experimental evaluation of a new sel-expanding patent ductus arteriosus in a canine model. J Vasc Interv Radiol 1996; 7: 877-87.

2. Masura J, Walsh KP, Thanopoulous B, et al. Catheter closure of moderate-to largesized patent ductus arteriosus using the new Amplatzer duct occluder: immediate and short term results. J Am Coll Cardiol 1998; 31: 878-82.

3. Thanoppoulos BD, Hakim FA, Hiari A, et al. Further experience with transcatheter closure of the patent ductus arteriosus using the Amplatzer duct occluder. J Am Coll Cardiol 2000; 35: 1016-21.

4. Simões LC, Pedra CAC, Esteves CA, et al. Fechamento percutâneo do canal arterial com a prótese Amplatzer: Experiência no Brasil. Arq Bras Cardiol, 2001; 77: 526-31

5. Faella HJ, Hijazi ZM. Closure of the patent ductus arteriosus with the Amplatzer PDA device: immediate results of the international clinical trial. Catheter Cardiovasc Interv 2000; 51: 50-4.

6. Tofeig M, Walsh KP, Arnold R. Transcatheter occlusion of a post-Fontan residual hepatic vein to pulmonary venous atrium communication using the Amplatzer septal occluder. Heart 1998; 79: 624-6.

7. Pedra CAC, Pihkala J, Nykanen DG, Benson LN. Antegrade transcatheter closure of coronary artery fistulae using vascular occlusion devices. Heart 2000; 83: 94-6.

8. Chessa M, Chaudhari M, DeGiovanni JV. Aorto-left ventricular tunnel: transcatheter closure using an Amplatzer duct occluder device. Am J Cardiol 2000; 86: 253-4

9. Wong PC, Barlow CF, Hickey PR, et al. Factors associated with choreoathetosis after cardiopulmonary bypass in children with congenital heart disease. Circulation 1992; 86: III18-II26.

10. Castaneda AR, Jonas RA, Mayer JE, Hanley FL. Tetralogy of Fallot. In: Castaned a AR, Jonas RA, Mayer JE, Hanley FL. Cardiac Surgery of the Neonate and Infant. Philadelphia: WB Saunders Co., 1994: 228.

11. Perry SB, Radhke W, Fellows KE, Keane JF, Lock JE. Coil embolization to occlude aortopulmonary collaterals vessels and shunts in patients with congenital heart disease. J Am Coll Cardiol 1989; 13: 100-8.

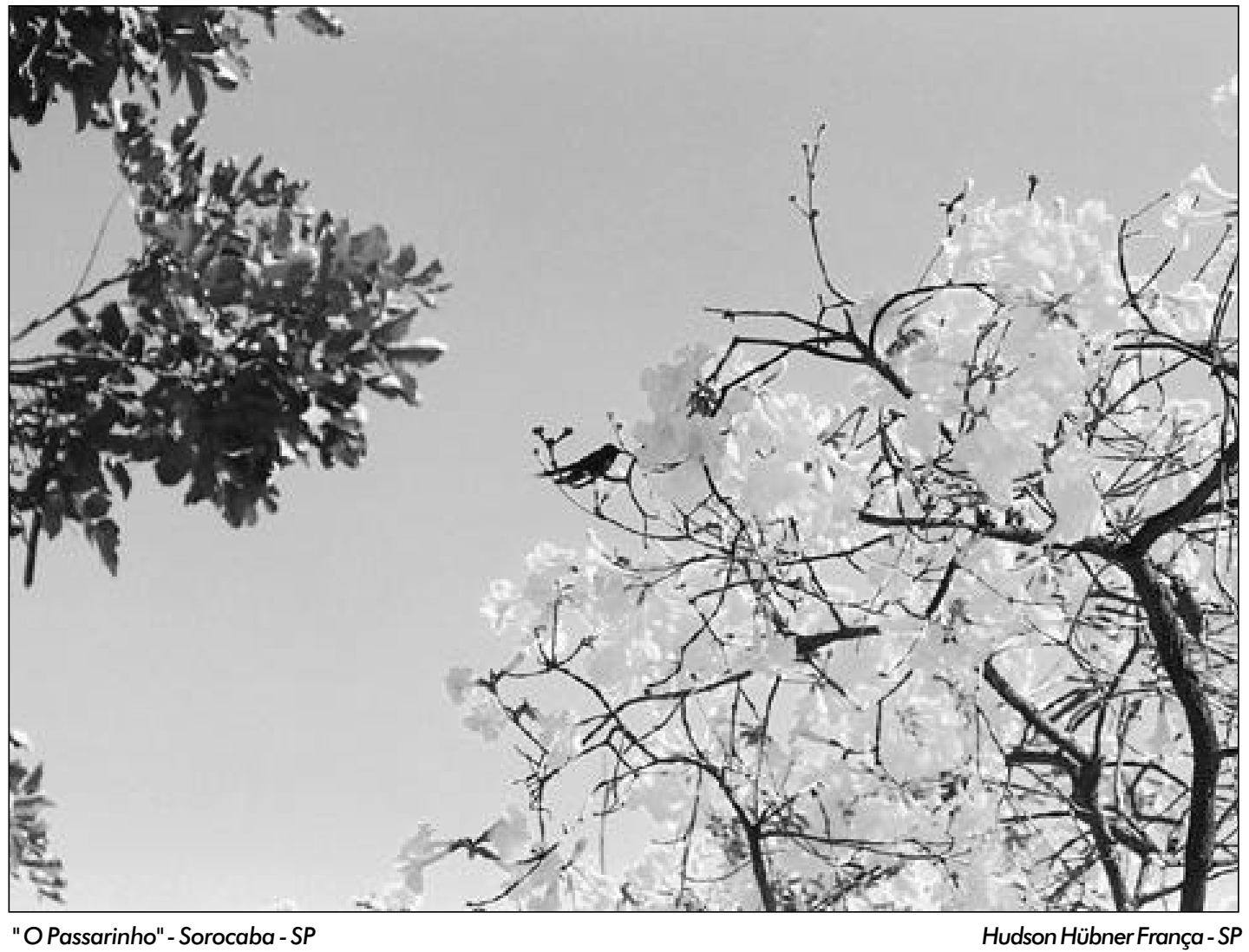

Editor da Secão de Fotografias Artísticas: Cícero Piva de Albuquerque

Correspondência: InCor - Av. Dr. Enéas C. Aguiar, 44 - 05403-000 - São Paulo, SP - E-mail: delcicero@incor. usp.br 